\title{
P2X4 receptors and neuropathic pain
}

\section{Makoto Tsuda, Takahiro Masuda, Hidetoshi Tozaki-Saitoh and Kazuhide Inoue*}

Department of Molecular and System Pharmacology, Graduate School of Pharmaceutical Sciences, Kyushu University, Fukuoka, Japan

\section{Edited by:}

Geoffrey Burnstock, University College

London Medical School, UK

Reviewed by:

Michael W. Salter, Smyth Medical

Centre, Canada

Francois Rassendren, Centre National

de la Recherche Scientifique, France

*Correspondence:

Kazuhide Inoue, Department of Molecular and System Pharmacology,

Graduate School of Pharmaceutical

Sciences, Kyushu University, 3-1-1

Maidashi, Higashi-ku, Fukuoka

812-8582, Japan

e-mail: inoue@phar.kyushu-u.ac.jp
Neuropathic pain, a debilitating pain condition, is a common consequence of damage to the nervous system. Neuropathic pain is often resistant to currently available analgesics. A growing body of evidence indicates that spinal microglia react and undergo a series of changes that directly influence the establishment of neuropathic pain states. After nerve injury, P2X4 receptors (P2X4Rs) are upregulated in spinal microglia by several factors at the transcriptional and translational levels. Those include the CC chemokine CCL21 derived from damaged neurons, the extracellular matrix protein fibronectin in the spinal cord, and the transcription factor interferon regulatory factor 8 (IRF8) expressed in microglia. P2X4R expression in microglia is also regulated at the post-translational level by signaling from other cell-surface receptors such as CC chemokine receptor (CCR2). Importantly, inhibiting the function or expression of P2X4Rs and P2X4R-regulating molecules suppresses the aberrant excitability of dorsal horn neurons and neuropathic pain. These findings indicate that P2X4R-positive microglia are a central player in mechanisms for neuropathic pain. Thus, microglial P2X4Rs are a potential target for treating the chronic pain state.

\section{Keywords: P2X4 receptor, microglia, neuropathic pain, spinal cord, fibronectin, CCL21, IRF8, BDNF}

\section{INTRODUCTION}

Injury to the nervous system arising from bone compression in cancer, diabetes mellitus, infection, autoimmune diseases, or traumatic injury results in debilitating chronic pain states (socalled neuropathic pain). Characteristic symptoms of neuropathic pain include spontaneous pain, hyperalgesia (increased pain perception of noxious stimuli), and tactile allodynia (pain hypersensitivity to normally innocuous stimuli). Neuropathic pain is refractory to currently available treatments, such as non-steroidal anti-inflammatory drugs and opioids (Costigan et al., 2009). Neuropathic pain is now considered not just a symptom of disease but also the consequence of disordered functioning of the nervous system (Costigan et al., 2009; Beggs et al., 2012).

Extensive lines of evidence from basic pain research using diverse animal models of neuropathic pain indicated that neuropathic pain is a reflection of the aberrant excitability of dorsal horn neurons evoked by peripheral sensory inputs (Woolf and Salter, 2000; Costigan et al., 2009). While neurons have long been considered the only cell type involved in neuropathic pain, recent studies have shown that pathologically altered neurotransmission requires communication with glial cells, in particular microglia, activated in the spinal cord in response to peripheral nerve injury (PNI; Watkins et al., 2001; Tsuda et al., 2005; McMahon and Malcangio, 2009; Ren and Dubner, 2010; Tsuda et al., 2013).

Microglial cells are resident macrophages in the central nervous system (CNS), which derive from primitive macrophages in the yolk sac (Ginhoux et al., 2010). In the adult, microglia are ubiquitously distributed throughout the brain and spinal cord and have small cell body bearing branched and motile processes, which monitor the local environment in the CNS
(Davalos et al., 2005; Nimmerjahn et al., 2005). Microglia show a stereotypical long-term response to a wide range of stimuli that threaten physiological homeostasis, including PNI. In response to PNI, microglia activation in the spinal cord progresses through hypertrophic morphology, an increase in cell number, and altered gene expression (Tsuda et al., 2005; Suter et al., 2007; Tsuda et al., 2009b). Activated microglia induce or enhance expression of various genes including neurotransmitter receptors such as purinergic P2 receptors (Pocock and Kettenmann, 2007). By responding to extracellular stimuli such as ATP, activated glia evoke various cellular responses such as production and release of bioactive factors including cytokines and neurotrophic factors (Inoue, 2006), which in turn leads to hyperexcitability of dorsal horn neurons and neuropathic pain.

Among purinergic $\mathrm{P} 2$ receptors [ionotropic receptors (P2XRs) and metabotropic receptors (P2YRs)], activated microglia express several subtypes of P2XRs and P2YRs, and these receptors play a key role in establishing and maintaining neuropathic pain states (Tsuda et al., 2012, 2013). In this article, we highlight recent advances that further increase our understanding of the mechanisms underlying neuropathic pain, with a specific focus on P2X4 receptor (P2X4R) in spinal microglia after PNI.

\section{P2X4R IN SPINAL MICROGLIA AND NEUROPATHIC PAIN}

The first observation that demonstrated the important role of $\mathrm{P} 2 \mathrm{X} 4 \mathrm{R}$ in neuropathic pain was that established tactile allodynia after PNI was reversed by pharmacological blockade of P2X4Rs in the spinal cord (Tsuda et al., 2003). Immunohistochemical studies revealed that expression of P2X4Rs in the spinal cord was 
upregulated exclusively in microglia. These results indicated that PNI-induced pain hypersensitivity depended on ongoing signaling via microglial P2X4Rs. Furthermore, animals with P2X4R knock-down or knock-out in the spinal cord were resistant to PNI-induced tactile allodynia (Tsuda et al., 2003; Ulmann et al., 2008; Tsuda et al., 2009a), indicating a necessity for P2X4Rs. The impact of microglial P2X4R stimulation in transforming tactile information to pain was demonstrated by an in vivo microglia transfer approach (Tsuda et al., 2003). It was found that intrathecal delivery of P2X4R-stimulated microglia caused normal rats to develop allodynia and indicated that microglial P2X4R stimulation is sufficient (Tsuda et al., 2003, 2005). Furthermore, it was demonstrated that activation of microglial P2X4Rs stimulated the synthesis and release of brain-derived neurotrophic factor (BDNF; Ulmann et al., 2008; Trang et al., 2009) and that BDNF then causes an altered transmembrane anion gradient in a subpopulation of dorsal horn lamina I neurons presumably through the downregulation of the neuronal chloride transporter KCC2, which in turn renders GABA and glycine effects depolarizing, rather than hyperpolarizing, in these neurons (Coull et al., 2005; Figure 1). Thus, P2X4R-stimulated microglia release BDNF as a crucial factor to signal lamina I neurons, causing an aberrant nociceptive output that contributes to neuropathic pain (Beggs et al., 2012). Therefore, microglial P2X4Rs are central players in the pathogenesis of neuropathic pain.

\section{REGULATION OF P2X4R EXPRESSION IN MICROGLIA: TRANSCRIPTIONAL, TRANSLATIONAL, AND POST-TRANSLATIONAL LEVELS}

Upregulation of $\mathrm{P} 2 \mathrm{X} 4 \mathrm{R}$ expression in microglia is a key process in the pathogenesis of neuropathic pain. Several studies have identified molecules involved in the upregulation of P2X4R expression in spinal microglia after PNI.

A clue to identifying an inducer of $\mathrm{P} 2 \mathrm{X} 4 \mathrm{R}$ expression in microglia was that microglial $\mathrm{P} 2 \mathrm{X} 4 \mathrm{R}$ upregulation was observed following PNI but not peripheral tissue inflammation (Tsuda et al., 2003), raising the possibility that a factor derived from injured primary afferent sensory neurons might be involved. Recently, it was shown that the chemokine CCL21 [chemokine (C-C motif) ligand 21] was induced in injured dorsal root ganglion (DRG) neurons and transported to the central terminals of the dorsal horn (Biber et al., 2011). Mice treated with CCL21-neutralizing antibody and mice deficient for CCL21 showed attenuation of tactile allodynia and microglial P2X4R upregulation. CCL21 treatment increased the expression of P2X4R in cultured microglia, indicating a direct action of CCL21 on microglia. Intrathecal supply of CCL21 in CCL21-deficient mice rescued PNI-induced tactile allodynia in those mice. Thus, CCL21 derived from injured DRG neurons directly contributes to microglial P2X4R expression and neuropathic pain (Biber et al., 2011; Figure 1).

Because blood-spinal cord barrier functions collapse after PNI occurs (Beggs et al., 2010; Echeverry et al., 2011), proteins leaking from the blood might change P2X4R expression in microglia. The extracellular matrix protein fibronectin might be such a protein. The level of fibronectin protein was elevated in the dorsal horn after PNI (Nasu-Tada et al., 2006; Echeverry et al., 2011).
Fibronectin stimulation induces upregulation of P2X4R mRNA and protein in primary cultured microglial cells (Nasu-Tada et al., 2006). Using integrin blockers in vitro and in vivo, it was shown that fibronectin/integrin signaling was crucial for augmentation of P2X4R expression and PNI-induced tactile allodynia (Tsuda et al., 2008a). Furthermore, intrathecal injection of fibronectin to naïve animals produced tactile allodynia, a behavior not observed in P2X4R-deficient mice administered fibronectin (Tsuda et al., 2008a). Regarding intracellular signaling mechanisms underlying P2X4R upregulation by fibronectin, microglial Lyn tyrosine kinase, a member of Src-family kinases (SFKs) that belong to the non-receptor protein tyrosine kinase family, is an important molecule as upregulation of P2X4R gene expression in response to fibronectin is not observed in microglial cells lacking Lyn (Tsuda et al., 2008b). In spinal cord microglial cells, Lyn was the predominant SFK (Tsuda et al., 2008b) amongst the five members (Src, Fyn, Lck, Yes, and Lyn) that are known to be expressed in the CNS (Salter and Kalia, 2004). Lyn expression in the spinal cord in vivo is highly restricted to microglia, and following PNI the level of Lyn is increased (Tsuda et al., 2008b), which is interferon- $\gamma$ signaling dependent (Tsuda et al., 2009b). Mice lacking Lyn suppress PNIinduced tactile allodynia and the upregulation of spinal P2X4R expression after PNI (Tsuda et al., 2008b). Following Lyn tyrosine kinase activation, two intracellular signaling cascades are distinctly activated: one is a pathway through phosphatidylinositol 3-kinase (PI3K)-Akt and the other is through mitogen-activated protein kinase kinase (MAPK kinase, MEK)-extracellular signalregulated kinase (ERK; Tsuda et al., 2009c). Signaling through the PI3K-Akt pathway induced degradation of p53 via mouse double minute 2 in a proteasome-dependent manner. The consequence of an attenuated repressive effect of p53 may be associated with enhanced P2X4 gene expression. However, activated MEK-ERK signaling in microglia exposed to fibronectin enhanced eukaryotic translation initiation factor 4E (eIF4E) phosphorylation status via activated MAPK-interacting protein kinase-1, which may play a role in regulating $\mathrm{P} 2 \mathrm{X} 4$ expression at translational levels (Figure 1). The Lyn-ERK signaling pathway seems likely to be active in spinal microglia after PNI as pharmacological inhibition of SFK effectively suppressed ERK activity in spinal microglia (Katsura et al., 2006). Thus, Lyn might be a key kinase in the molecular machinery mediating the upregulation of P2X4R in microglia (Figure 1).

To detect extracellular ATP, P2X4Rs are expressed on the cell surface of microglia. However, a large amount of P2X4R protein within microglia (and macrophages) localizes predominantly to intracellular lysosomal compartments (Qureshi et al., 2007). Interestingly, $\mathrm{P} 2 \mathrm{X} 4 \mathrm{R}$ protein remains stable within the proteolytic environment of lysosomes. How P2X4R protein is recruited to the cell surface of microglia remains elusive, but recent studies have shown that trafficking of $\mathrm{P} 2 \mathrm{X} 4 \mathrm{R}$ protein to the cell surface occurs when microglia are stimulated by a Tolllike receptor (TLR) 4 agonist lipopolysaccharide (Boumechache et al., 2009; Toulme et al., 2010) or the $\mathrm{Ca}^{2+}$ ionophore, ionomycin (Qureshi et al., 2007). Furthermore, the chemokine CCL2 increased P2X4R protein levels on the cell surface (without changing total cellular expression) via CC chemokine receptor (CCR2) (Toyomitsu et al., 2012). Notably, CCL2 changed the distribution 


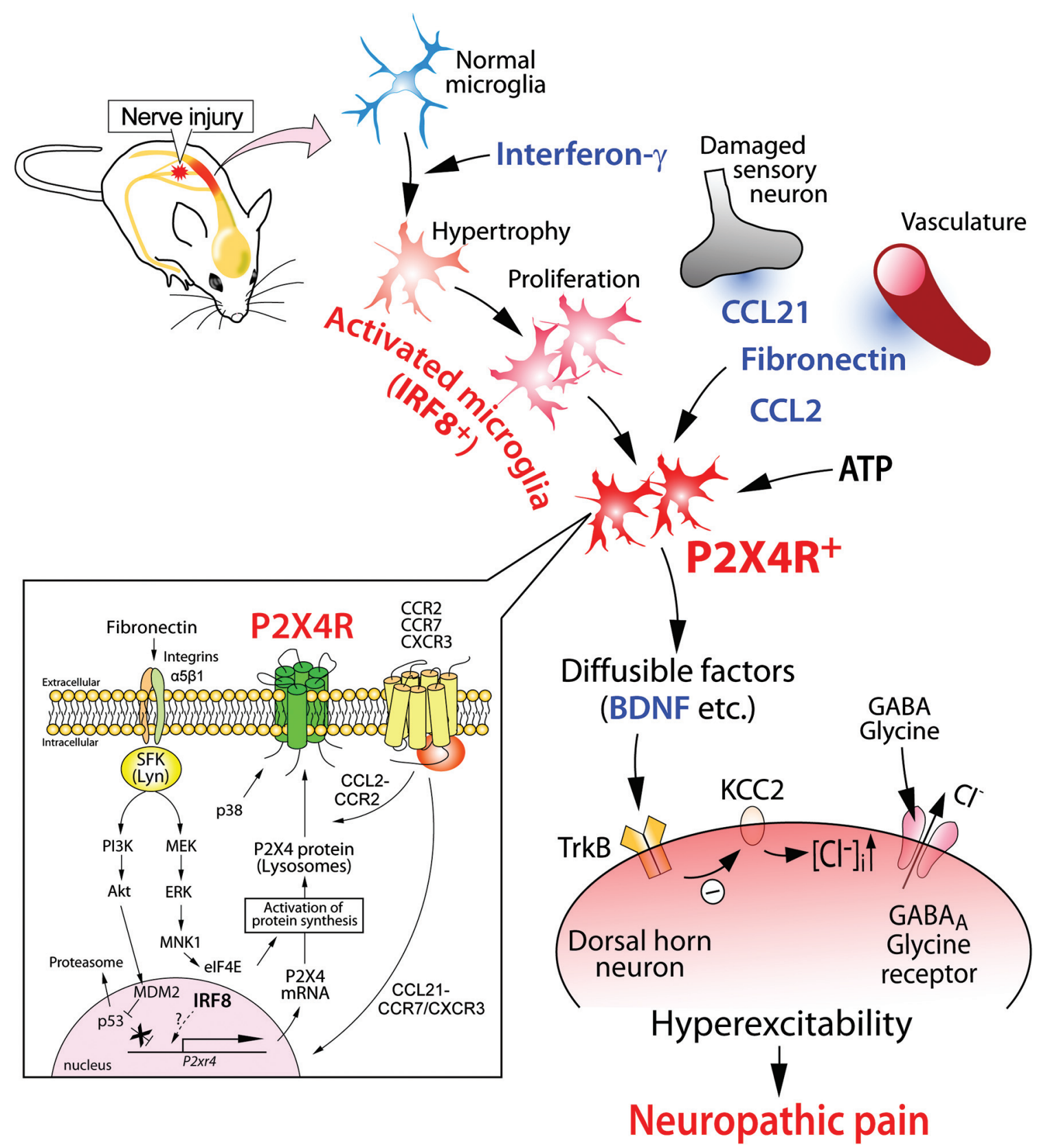

FIGURE 1 | Schematic illustration of the potential mechanisms by which P2X4R in activated microglia modulate pain signaling in the dorsal horn after PNI. Nerve injury activates microglia in the dorsal horn of the spinal cord. Activated microglia show increased expression of P2X4R. The upregulation of microglial P2X4R expression involves signaling by fibronectin and chemokine (C-C motif) ligand 21 (CCL21). CCL2 signaling promotes P2X4R trafficking to cell surface of microglia. P2X4R is activated by ATP and, in turn, release bioactive diffusible factors, such as BDNF. BDNF downregulates the potassium-chloride transporter KCC2 via TrkB, causes an increase in intracellular $\left[\mathrm{Cl}^{-}\right]$, and leads to the collapse of the transmembrane anion gradient in dorsal horn neurons which in turn induces depolarization of these neurons following stimulation by GABA and glycine. The resultant hyperexcitability in the dorsal horn pain network induced by factors from activated microglia may be responsible for neuropathic pain. of lysosomes with P2X4R protein within microglial cells and induced the release of a lysosomal enzyme (Toyomitsu et al., 2012). Thus, CCL2 might promote the expression of P2X4R protein on the cell surface of microglia through exocytosis of P2X4R-containing lysosomes (Figure 1). A recent study using single-molecule imaging to track P2X4Rs in the processes of microglia showed that lateral mobility of P2X4Rs is enhanced in activated microglia by the p38 MAPK pathway that selectively regulates slowly mobile P2X4Rs (Toulme and Khakh, 2012). These results indicated that microglial P2X4Rs are dynamically 
regulated on the cell surface (Figure 1). Thus, post-translational regulation to enhance $\mathrm{P} 2 \mathrm{X} 4 \mathrm{R}$ expression and mobility on the cell surfaces of microglia might render cells hyper-responsive to extracellular ATP, which may be important in neuropathic pain.

\section{TRANSCRIPTIONAL FACTOR FOR REACTIVE STATES OF MICROGLIA}

PNI activates microglia and converts them to reactive phenotypes through the activation of gene transcription. For example, as described above, activated microglia with high levels of P2X4R expression are essential for PNI-induced neuropathic pain. Appropriate changes in gene expression patterns required for transformation into reactive phenotypes might be tightly regulated by cell type-specific transcription factors. Recent studies identified interferon regulatory factor 8 (IRF8) as a transcription factor in microglia (Horiuchi et al., 2012; Masuda et al., 2012; Minten et al., 2012; Kierdorf et al., 2013) that was critical for their activation and neuropathic pain (Masuda et al., 2012). IRF8 is a member of the IRF family (IRF1-9), and is expressed in immune cells such as lymphocytes and dendritic cells (Tamura et al., 2008). Within the spinal cord, IRF8 expression was markedly upregulated in microglia, but not in neurons or astrocytes, after PNI (Masuda et al., 2012). The microglia-specific upregulation of IRF8 occurs as early as postoperative day 1 , peaks on day 3 , and persists for at least several weeks. IRF8-deficient mice showed a reduction of PNIinduced tactile allodynia with no change in basal mechanical sensitivity. Notably, suppressing the upregulated expression of spinal IRF8 after PNI by intrathecal administration of a small interfering RNA (siRNA) targeting IRF8 in wild-type mice with allodynia caused a significant recovery of tactile allodynia. This indicated an ongoing activation of IRF8 in spinal microglia. In vitro and in vivo studies demonstrated that IRF8 promoted the transcription of $\mathrm{P} 2 \mathrm{X} 4 \mathrm{R}$ and other molecules associated with reactive phenotypes [innate immune response (TLR2), chemotaxis (P2Y12R and the chemokine receptor CX3CR1), and inflammatory components (interleukin- $\beta$, cathepsin $\mathrm{S}$, and BDNF)]. Nevertheless, nerve injury-induced proliferation of spinal microglia was not affected by IRF8 deficiency, indicating IRF8 deficiency does not impair all reactive processes of microglia. Rather the transcription factor contributes to determining the reactive phenotypes of microglia by changing the expression of a set of genes including P2X4R (Figure 1). However, several important issues remain. How is IRF8 expression induced in microglia after PNI? To determine whether IRF8 directly binds to promoter regions of these genes is also important. Answers to these issues will provide new insights into the molecular mechanisms underlying microglial activation and neuropathic pain.

\section{REFERENCES}

Beggs, S., Liu, X. J., Kwan, C., and Salter, M. W. (2010). Peripheral nerve injury and TRPV1-expressing primary afferent C-fibers cause opening of the blood-brain barrier. Mol. Pain 6:74. doi: 10.1186/17448069-6-74

\section{MARKERS OF ACTIVATED MICROGLIA IN VIVO}

A widely used index for predicting the status of microglia activity in vivo is the expression levels of microglial markers [complement receptor 3 (CR3, recognized by OX-42) and Iba1]. Increased immunolabeling of these markers is a principal feature of a variety of PNI models (Tsuda et al., 2005; Suter et al., 2007), but it remains unclear whether their expression can be linked to tactile allodynia following PNI as in some cases CR3 expression is not well correlated with the degree of allodynia (Colburn et al., 1997; Winkelstein and DeLeo, 2002; Tsuda et al., 2005; Suter et al., 2007). In addition, OX-42 immunofluorescence was still observed in P2X4R-knockdown animals that had attenuated PNIinduced allodynia (Tsuda et al., 2003). In contrast, as described above, microglia-specific molecules including P2X4R and IRF8 are necessary for the pathology of neuropathic pain, in both its development and maintenance phases. Therefore, alterations in the expression or phosphorylation of these molecules are likely to be more useful markers to assess reactive states of microglia in vivo.

\section{CONCLUDING REMARKS}

We have primarily focused on the role of P2X4R expressed in spinal microglia in neuropathic pain (Figure 1). Pharmacological, molecular, and genetic studies on P2X4Rs described above provide compelling evidence that $\mathrm{P} 2 \mathrm{X} 4 \mathrm{R}$-positive microglia are a central player in the mechanisms of neuropathic pain and might be promising targets for treating neuropathic pain. Furthermore, the upregulation of microglial P2X4R expression has also been reported in animal models of stroke (Cavaliere et al., 2003), brain tumor (Guo et al., 2004), traumatic brain injury (Guo and Schluesener, 2005; Zhang et al., 2007), spinal cord injury (Schwab et al., 2005), epilepsy (Ulmann et al., 2013), and in human acute inflammatory demyelinating polyradiculoneuropathy (Zhang et al., 2008). Recently, it was demonstrated that P2X4R-positive microglia are essential for morphine-induced hyperalgesia via a P2X4R-BDNF-KCC2 disinhibition cascade between microglia and dorsal horn neurons (Ferrini et al., 2013). It is expected that an increased understanding of the phenotype of P2X4Rpositive microglia will provide us with exciting insights into pain mechanisms and clues to aid the development of new therapeutic agents for the management of neuropathic pain and other CNS diseases.

\section{ACKNOWLEDGMENTS}

This work was supported by grants from the JSPS through the NEXT Program initiated by the CSTP (Makoto Tsuda) and the MEXT of Japan (Takahiro Masuda, Makoto Tsuda, Kazuhide Inoue), and from Core Research for Evolutional Science and Technology of JST (Kazuhide Inoue).

expression and initiates neuropathic pain development. EMBO J. 30, 1864-1873. doi: 10.1038/emboj. 2011.89

Boumechache, M., Masin, M., Edwardson, J. M., Gorecki, D. C., and Murrell-Lagnado, R. (2009). Analysis of assembly and trafficking of native $\mathrm{P} 2 \mathrm{X} 4$ and $\mathrm{P} 2 \mathrm{X} 7$ receptor complexes in rodent immune cells. J. Biol. Chem. 284, 13446-13454. doi: 10.1074/jbc.m901255200

Cavaliere, F., Florenzano, F., Amadio, S., Fusco, F. R., Viscomi, M. T., D'Ambrosi, N., et al. (2003). Upregulation of $\mathrm{P} 2 \mathrm{X} 2, \mathrm{P} 2 \mathrm{X} 4$ receptor 
and ischemic cell death: prevention by $\mathrm{P} 2$ antagonists. Neuroscience 120, 85-98. doi: 10.1016/s03064522(03)00228-8

Colburn, R. W., DeLeo, J. A., Rickman, A. J., Yeager, M. P., Kwon, P., and Hickey, W. F. (1997). Dissociation of microglial activation and neuropathic pain behaviors following peripheral nerve injury in the rat. J. Neuroimmunol. 79, 163 175. doi: 10.1016/s0165-5728(97) 00119-7

Costigan, M., Scholz, J., and Woolf, C. J. (2009). Neuropathic pain: a maladaptive response of the nervous system to damage. Annu. Rev. Neurosci. 32, 1-32. doi: 10. 1146/annurev.neuro.051508.135531

Coull, J. A., Beggs, S., Boudreau, D., Boivin, D., Tsuda, M., Inoue, K., et al. (2005). BDNF from microglia causes the shift in neuronal anion gradient underlying neuropathic pain. Nature 438, 1017-1021. doi: $10.1038 /$ nature 04223

Davalos, D., Grutzendler, J., Yang, G., Kim, J. V., Zuo, Y., Jung, S., et al. (2005). ATP mediates rapid microglial response to local brain injury in vivo. Nat. Neurosci. 8, 752758. doi: $10.1038 / \mathrm{nn} 1472$

Echeverry, S., Shi, X. Q., Rivest, S., and Zhang, J. (2011). Peripheral nerve injury alters blood-spinal cord barrier functional and molecular integrity through a selective inflammatory pathway. $J$. Neurosci. 31, 10819-10828. doi: 10. 1523/jneurosci.1642-11.2011

Ferrini, F., Trang, T., Mattioli, T. A., Laffray, S., Del'guidice, T., Lorenzo, L. E., et al. (2013). Morphine hyperalgesia gated through microgliamediated disruption of neuronal $\mathrm{Cl}(-)$ homeostasis. Nat. Neurosci. 16, 183-192. doi: 10.1038/nn.3295

Ginhoux, F., Greter, M., Leboeuf, M., Nandi, S., See, P., Gokhan, S., et al. (2010). Fate mapping analysis reveals that adult microglia derive from primitive macrophages. Science 330, 841-845. doi: 10. 1126/science.1194637

Guo, L. H., and Schluesener, H. J. (2005). Lesional accumulation of P2X(4) receptor(+) macrophages in rat CNS during experimental autoimmune encephalomyelitis. Neuroscience 134, 199-205. doi: 10. 1016/j.neuroscience.2005.04.026

Guo, L. H., Trautmann, K., and Schluesener, H. J. (2004). Expression of $\mathrm{P} 2 \mathrm{X} 4$ receptor in rat C6 glioma by tumor-associated macrophages and activated microglia. J. Neuroimmunol. 152, 67-72. doi: 10.1016/j. jneuroim.2004.04.005
Horiuchi, M., Wakayama, K., Itoh, A., Kawai, K., Pleasure, D., Ozato, K., et al. (2012). Interferon regulatory factor $8 /$ interferon consensus sequence binding protein is a critical transcription factor for the physiological phenotype of microglia. J. Neuroinflammation 9:227. doi: 10. 1186/1742-2094-9-227

Inoue, K. (2006). The function of microglia through purinergic receptors: neuropathic pain and cytokine release. Pharmacol. Ther. 109, 210-226. doi: 10.1016/j. pharmthera.2005.07.001

Katsura, H., Obata, K., Mizushima, T., Sakurai, J., Kobayashi, K., Yamanaka, H., et al. (2006). Activation of Src-family kinases in spinal microglia contributes to mechanical hypersensitivity after nerve injury. J. Neurosci. 26, 8680-8690. doi: 10.1523/jneurosci. 1771-06.2006

Kierdorf, K., Erny, D., Goldmann, T., Sander, V., Schulz, C., Perdiguero, E. G., et al. (2013). Microglia emerge from erythromyeloid precursors via Pu.1- and Irf8-dependent pathways. Nat. Neurosci. 16, 273-280. doi: 10. 1038/nn.3318

Masuda, T., Tsuda, M., Yoshinaga, R., Tozaki-Saitoh, H., Ozato, K., Tamura, T., et al. (2012). IRF8 is a critical transcription factor for transforming microglia into a reactive phenotype. Cell Rep. 1, 334-340. doi: 10.1016/j.celrep.2012.02.014

McMahon, S. B., and Malcangio, M. (2009). Current challenges in gliapain biology. Neuron 64, 46-54. doi: 10.1016/j.neuron.2009.09.033

Minten, C., Terry, R., Deffrasnes, C., King, N. J. C., and Campbell, I. L. (2012). IFN regulatory factor 8 is a key constitutive determinant of the morphological and molecular properties of microglia in the CNS. PLoS One 7:e49851. doi: 10.1371/journal. pone.0049851

Nasu-Tada, K., Koizumi, S., Tsuda, M., Kunifusa, E., and Inoue, K. (2006). Possible involvement of increase in spinal fibronectin following peripheral nerve injury in upregulation of microglial P2X(4), a key molecule for mechanical allodynia. Glia 53, 769-775. doi: 10.1002/glia.20339

Nimmerjahn, A., Kirchhoff, F., and Helmchen, F. (2005). Resting microglial cells are highly dynamic surveillants of brain parenchyma in vivo. Science 308, 1314-1318. doi: 10.1126/science. 1110647

Pocock, J. M., and Kettenmann, H. (2007). Neurotransmitter receptors on microglia. Trends Neurosci. 30,
527-535. doi: 10.1016/j.tins.2007. 07.007

Qureshi, O. S., Paramasivam, A., Yu, J. C., and Murrell-Lagnado, R. D. (2007). Regulation of P2X4 receptors by lysosomal targeting, glycan protection and exocytosis. J. Cell Sci. 120, 3838-3849. doi: 10.1242/jcs. 010348

Ren, K., and Dubner, R. (2010). Interactions between the immune and nervous systems in pain. Nat. Med. 16, 1267-1276. doi: $10.1038 / \mathrm{nm}$. 2234

Salter, M. W., and Kalia, L. V. (2004). Src kinases: a hub for NMDA receptor regulation. Nat. Rev. Neurosci. 5, 317-328. doi: 10.1038/nrn1368

Schwab, J. M., Guo, L., and Schluesener, H. J. (2005). Spinal cord injury induces early and persistent lesional P2X4 receptor expression. J. Neuroimmunol. 163, 185-189. doi: 10. 1016/j.jneuroim.2005.02.016

Suter, M. R., Wen, Y. R., Decosterd, I., and Ji, R. R. (2007). Do glial cells control pain? Neuron Glia Biol. 3, 255-268. doi: 10. 1017/S1740925X08000100

Tamura, T., Yanai, H., Savitsky, D., and Taniguchi, T. (2008). The IRF family transcription factors in immunity and oncogenesis. Аnnu. Rev. Immunol. 26, 535-584. doi: 10. 1146/annurev.immunol.26.021607. 090400

Toulme, E., Garcia, A., Samways, D., Egan, T. M., Carson, M. J., and Khakh, B. S. (2010). P2X4 receptors in activated C8-B4 cells of cerebellar microglial origin. J. Gen. Physiol. 135, 333-353. doi: 10.1085/jgp. 200910336

Toulme, E., and Khakh, B. S. (2012). Imaging P2X4 receptor lateral mobility in microglia regulation by calcium and p38 MAPK. J. Biol. Chem. 287, 14734-14748. doi: 10. 1074/jbc.m111.329334

Toyomitsu, E., Tsuda, M., Yamashita, T., Tozaki-Saitoh, H., Tanaka, Y., and Inoue, K. (2012). CCL2 promotes $\mathrm{P} 2 \mathrm{X} 4$ receptor trafficking to the cell surface of microglia. Purinergic Signal. 8, 301-310. doi: 10. 1007/s11302-011-9288-x

Trang, T., Beggs, S., Wan, X., and Salter, M. W. (2009). P2X4-receptormediated synthesis and release of brain-derived neurotrophic factor in microglia is dependent on calcium and p38-mitogenactivated protein kinase activation. J. Neurosci. 29, 3518-3528. doi: 10. 1523/JNEUROSCI.5714-08.2009

Tsuda, M., Inoue, K., and Salter, M. W. (2005). Neuropathic pain and spinal microglia: a big problem from molecules in "small" glia. Trends Neurosci. 28, 101-107. doi: 10. 1016/j.tins.2004.12.002

Tsuda, M., Kuboyama, K., Inoue, T., Nagata, K., Tozaki-Saitoh, H., and Inoue, K. (2009a). Behavioral phenotypes of mice lacking purinergic P2X4 receptors in acute and chronic pain assays. Mol. Pain 5:28. doi: 10 . 1186/1744-8069-5-28

Tsuda, M., Masuda, T., Kitano, J., Shimoyama, H., Tozaki-Saitoh, H., and Inoue, K. (2009b). IFN-gamma receptor signaling mediates spinal microglia activation driving neuropathic pain. Proc. Natl. Acad. Sci. U S A 106, 8032-8037. doi: 10. 1073/pnas.0810420106

Tsuda, M., Masuda, T., Tozaki-Saitoh, H., and Inoue, K. (2013). Microglial regulation of neuropathic pain. $J$. Pharmacol. Sci. 121, 89-94. doi: 10. 1254/jphs.12r14cp

Tsuda, M., Shigemoto-Mogami, Y., Koizumi, S., Mizokoshi, A., Kohsaka, S., Salter, M. W., et al. (2003). P2X4 receptors induced in spinal microglia gate tactile allodynia after nerve injury. Nature 424, 778-783. doi: 10. 1038/nature01786

Tsuda, M., Toyomitsu, E., Komatsu, T., Masuda, T., Kunifusa, E., Nasu-Tada, K., et al. (2008a). Fibronectin/integrin system is involved in $\mathrm{P} 2 \mathrm{X}(4)$ receptor upregulation in the spinal cord and neuropathic pain after nerve injury. Glia 56, 579-585. doi: 10.1002/glia. 20641

Tsuda, M., Toyomitsu, E., Kometani, M., Tozaki-Saitoh, H., and Inoue, K. (2009c). Mechanisms underlying fibronectin-induced upregulation of P2XR expression in microglia: distinct roles of PI3K-Akt and MEKERK signaling pathways. J. Cell. Mol. Med. 13, 3251-3259. doi: 10.1111/j. 1582-4934.2009.00719.x

Tsuda, M., Tozaki-Saitoh, H., and Inoue, K. (2012). Purinergic system, microglia and neuropathic pain. Curr. Opin. Pharmacol. 12, 74-79. doi: 10.1016/j.coph.2011.10.014

Tsuda, M., Tozaki-Saitoh, H., Masuda, T., Toyomitsu, E., Tezuka, T., Yamamoto, T., et al. (2008b). Lyn tyrosine kinase is required for $\mathrm{P} 2 \mathrm{X}(4)$ receptor upregulation and neuropathic pain after peripheral nerve injury. Glia 56, 50-58. doi: 10. 1002/glia.20591

Ulmann, L., Hatcher, J. P., Hughes, J. P., Chaumont, S., Green, P. J., Conquet, F., et al. (2008). Upregulation of $\mathrm{P} 2 \mathrm{X} 4$ receptors in spinal microglia after peripheral nerve injury mediates BDNF 
release and neuropathic pain. J. Neurosci. 28, 11263-11268. doi: $\quad 10.1523 /$ jneurosci.230808.2008

Ulmann, L., Levavasseur, F., Avignone, E., Peyroutou, R., Hirbec, H., Audinat, E., et al. (2013). Involvement of P2X4 receptors in hippocampal microglial activation after status epilepticus. Glia 61, 1306-1319. doi: 10.1002/glia. 22516

Watkins, L. R., Milligan, E. D., and Maier, S. F. (2001). Glial activation: a driving force for pathological pain. Trends Neurosci. 24, 450455. doi: 10.1016/s0166-2236(00) 01854-3

Winkelstein, B. A., and DeLeo, J. A. (2002). Nerve root injury severity differentially modulates spinal glial activation in a rat lumbar radiculopathy model: considerations for persistent pain. Brain Res. 956, 294 301. doi: 10.1016/s0006-8993(02) 03560-6

Woolf, C. J., and Salter, M. W. (2000). Neuronal plasticity: increasing the gain in pain. Science 288, 17651769. doi: 10.1126/science.288.5472. 1765

Zhang, Z., Zhang, Z., Artelt, M., Burnet, M., and Schluesener, H. J. (2007). Dexamethasone attenuates early expression of three molecules associated with microglia/macrophages activation following rat traumatic brain injury. Acta Neuropathol. doi: 10. 1007/s00401-007-0195-8
Zhang, Z., Zhang, Z. Y., Fauser, U., and Schluesener, H. J. (2008). Mechanical allodynia and spinal up-regulation of $\mathrm{P} 2 \mathrm{X} 4$ receptor in experimental autoimmune neuritis rats. Neuroscience 152, 495-501. doi: 10.1016/j.neuroscience.2007. 12.042

Conflict of Interest Statement: The authors declare that the research was conducted in the absence of any commercial or financial relationships that could be construed as a potential conflict of interest.

Received: 11 September 2013; accepted: 07 October 2013; published online: 28 October 2013.
Citation: Tsuda M, Masuda T, TozakiSaitoh $H$ and Inoue $K$ (2013) P2X4 receptors and neuropathic pain. Front. Cell. Neurosci. 7:191. doi: 10.3389/fncel.2013.00191

This article was submitted to the journal Frontiers in Cellular Neuroscience.

Copyright (c) 2013 Tsuda, Masuda, Tozaki-Saitoh and Inoue. This is an open-access article distributed under the terms of the Creative Commons Attribution License (CC BY). The use, distribution or reproduction in other forums is permitted, provided the original author(s) or licensor are credited and that the original publication in this journal is cited, in accordance with accepted academic practice. No use, distribution or reproduction is permitted which does not comply with these terms. 